\title{
OPEN Stabilization demands of walking modulate the vestibular contributions to gait
}

\begin{abstract}
Rina M. Magnani ${ }^{1}$, Sjoerd M. Bruijn ${ }^{2,3}$, Jaap H. van Dieën ${ }^{2}$ \& Patrick A. Forbes ${ }^{4 凶}$
Stable walking relies critically on motor responses to signals of head motion provided by the vestibular system, which are phase-dependent and modulated differently within each muscle. It is unclear, however, whether these vestibular contributions also vary according to the stability of the walking task. Here we investigate how vestibular signals influence muscles relevant for gait stability (medial gastrocnemius, gluteus medius and erector spinae)-as well as their net effect on ground reaction forces-while humans walked normally, with mediolateral stabilization, wide and narrow steps. We estimated local dynamic stability of trunk kinematics together with coherence of electrical vestibular stimulation (EVS) with muscle activity and mediolateral ground reaction forces. Walking with external stabilization increased local dynamic stability and decreased coherence between EVS and all muscles/ forces compared to normal walking. Wide-base walking also decreased vestibulomotor coherence, though local dynamic stability did not differ. Conversely, narrow-base walking increased local dynamic stability, but produced muscle-specific increases and decreases in coherence that resulted in a net increase in vestibulomotor coherence with ground reaction forces. Overall, our results show that while vestibular contributions may vary with gait stability, they more critically depend on the stabilization demands (i.e. control effort) needed to maintain a stable walking pattern.
\end{abstract}

The stability of walking is commonly assessed as the ability to maintain upright locomotion in the presence of self-generated and/or external perturbations ${ }^{1-3}$. To ensure stable walking, the nervous system relies on the integration and modulation of sensory signals from visual, somatosensory and vestibular sources to generate ongoing and/or corrective postural responses throughout the different phases of the gait cycle ${ }^{4-7}$. The vestibular system, for instance, which encodes signals of head movement in space $^{8}$, is assumed to contribute to gait stability because impaired gait is often observed in vestibulopathic patients ${ }^{9,10}$. Recent studies have further revealed that vestibular contributions to locomotion undergo phase- and muscle-specific responses that appear to align with each muscle's functional role in gait stability ${ }^{11,12}$. Specifically, the vestibular contributions to mediolateral stability during walking may be related to mediolateral foot placement produced by muscles around the hip ${ }^{11,13,14}$ and to ankle torque at push-off driven by muscles of the ankle ${ }^{15}$. In the sagittal plane, however, corrective responses to a vestibular disturbance are almost entirely absent throughout the gait cycle ${ }^{16}$. Because stability in the sagittal plane is maintained largely passively ${ }^{17}$ - due to passive dynamics of the legs ${ }^{18}$ - it may be possible that the control of whole-body stability during locomotion requires less feedback-driven control as compared to the frontal plane ${ }^{16}$. Thus, the question arises whether changes in the stability of walking in the mediolateral plane also influences the vestibular contribution to stability during locomotion. Therefore, our aim is to identify how vestibular contributions to balance are modulated across varying stabilization demands of walking by characterizing changes in coupling of vestibular input to motor outputs during externally-imposed and natural variations in stability.

Experimentally, gait stability can be improved by adding external lateral stabilization to the body, thereby removing the need for the nervous system to control upright balance in the mediolateral direction ${ }^{19-21}$. Under these stabilized conditions, step width variability, trunk and pelvis motion and premotor cortical involvement all decrease compared to normal walking ${ }^{22-25}$. These observations indicate a reduced need to actively control gait stability, and as a result, may also diminish the necessity for vestibular sensory feedback control. This may be similar to observations during upright standing, where compensatory responses to mediolaterally-directed vestibular disturbances are absent when participants are stabilized in the mediolateral direction, despite having

${ }^{1}$ Department of Physiotherapy, School of Physical Education and Physical Therapy, State University of Goiás, Goiânia, GO, Brazil. '2Department of Human Movement Sciences, Faculty of Behavioral and Movement Sciences, Vrije Universiteit Amsterdam, Amsterdam Movement Sciences, Amsterdam, The Netherlands. ${ }^{3}$ Institute Brain and Behavior Amsterdam, Amsterdam, The Netherlands. ${ }^{4}$ Department of Neuroscience, Erasmus MC, University Medical Center Rotterdam, P.O. Box 2040, 3000 CA Rotterdam, The Netherlands. ${ }^{\circledR}$ email: p.forbes@erasmusmc.nl 
to maintain balance in an anterior-posterior direction ${ }^{26}$. Therefore, we first hypothesized that external lateral stabilization of the body would diminish the muscle and whole-body responses to a mediolateral vestibular disturbance during locomotion.

Natural changes in gait are also thought to influence stability. For instance, adopting a wider step width has been described as a response to decreased lateral stability during locomotion ${ }^{22,27}$, and seems to be an effective approach to increase margins of stability (i.e. base of support) in older adults ${ }^{28,29}$. If the vestibular system influence on gait is tightly coupled to stability, then similar to external stabilization, we expected that the evoked muscle and whole-body responses to a vestibular disturbance should diminish when walking with wider steps. On the other hand, narrow-base walking requires increased effort to control gait stability in the frontal plane in young and old adults ${ }^{30,31}$, since the margins of stability, or tolerance for errors, are substantially reduced ${ }^{32,33}$. These observations, however, seem to contrast with measures of local dynamic stability, which quantify the likelihood of departing from a steady-state gait pattern in the absence of external disturbances, and instead indicate increased gait stability during narrow-base walking and decreased gait stability during wide-base walking ${ }^{31,34}$. We aim to test a potential explanation for this conflict by examining whether the increased local dynamic stability observed when walking with narrow step width is (partially) subserved by increased vestibular sensory feedback. Indeed, support for this is seen by the additional contribution of vestibular signals (via the lateral vestibular nuclei) to limb muscle activity in mice when walking on a narrow beam that is absent when walking on level ground ${ }^{35}$. Here, we tested these hypotheses by comparing muscle and whole-body responses evoked by a vestibular disturbance-together with estimates of stability-across normal, mediolaterally stabilized, widebase and narrow-base walking.

\section{Methods}

Participants. We measured 23 healthy young adults between 24 and 33 years recruited from the university campus. Twelve participants were excluded during data analysis due to technical problems in the collection of electromyography $(n=3)$ or kinematic data $(n=9)$ in any of the trials recorded. Here we present results from eleven young adults (four females, $28.5 \pm 2.9$ years old, $71.6 \pm 8.6 \mathrm{~kg}$ and $1.77 \pm 0.10 \mathrm{~m}$, body mass index $22.2 \pm 3.1 \mathrm{~kg} / \mathrm{m}^{2}$ ). Exclusion criteria included self-reported history of injury and/or disfunction of the nervous, musculoskeletal or vestibular systems, or the use of medications that can cause dizziness. Participants were also instructed not to participate in intense physical exercise on the day of the experiment. The participants agreed to participate in the study by signing the informed consent form, and the study was approved by the VU Amsterdam Research Ethics Committee (VCWE-2017-158). All methods were carried out in accordance with relevant guidelines and regulations.

Electrical vestibular stimulation. A continuous electrical vestibular stimulus (EVS) was used to deliver an isolated vestibular disturbance to participants during all walking trials. Coupling of the electrical stimulus with muscle activity and ground reaction forces was quantified over the gait cycle to determine the magnitude and timing of the vestibular contribution to ongoing muscle and whole-body responses. The electrical stimulus modulates the afferent firing rate of both semicircular canal and otolith afferents ${ }^{36-38}$, and when delivered in a binaural-bipolar configuration, EVS evokes a sensation of head roll rotational velocity ${ }^{39}$ about an axis directed posteriorly and superiorly by $18^{\circ}$ relative to the Reid plane $e^{40-42}$. When the head is facing forward, this stimulus configuration results in a postural response in the frontal plane to compensate for the induced roll error signal ${ }^{16,26,43-46}$.

The electrical stimulus was applied to participants using flexible carbon rubber electrodes $\left(9 \mathrm{~cm}^{2}\right)$. The electrodes were coated with Spectra 360 electrode gel (Parker La, USA) and fixed to participants' mastoid processes using adhesive tape and an elastic head band. The stimulus was delivered as an analog signal via a data acquisition board (National Instruments Corp., Austin, TX, USA) to an isolated constant current stimulator (STMISOLA, Biopac, Goleta, CA, USA). All participants were exposed to the same stochastic EVS designed with a limited bandwidth of 0 to $25 \mathrm{~Hz}^{47}$, zero-mean low-pass filtered white noise, $25 \mathrm{~Hz}$ cutoff, zero lag, fourthorder Butterworth, peak amplitude of $5.0 \mathrm{~mA}$, root mean square of $\sim 1.2 \mathrm{~mA}$, lasting $8 \mathrm{~min}$ and created with Matlab software (MathWorks, Natick, MA, USA). Because this binaural-bipolar stimulus oscillates around a zero-mean, the imposed sensations of roll motion, and the accompanying compensatory responses, occur in both a left and right direction.

Protocol. Participants walked on a dual-belt treadmill at a belt speed of $0.8 \mathrm{~m} / \mathrm{s}$ in four different conditions: normal walking, stabilized walking, wide-base walking, and narrow-base walking. During normal walking and stabilized walking, participants were instructed to walk with their naturally preferred step width. Stabilized walking was achieved using a custom-made spring-loaded mediolateral pelvic stabilization frame. The stabilization frame was attached through springs to two carts that allowed for movement in the anterior-posterior direction. The springs were pre-tensioned to provide a stabilizing stiffness of $1260 \mathrm{~N} / \mathrm{m}^{48}$ and the height of the carts was aligned with the height of the pelvis for each subject ${ }^{49}$. During wide-base walking, participants were instructed to increase their step width beyond the approximate width of their hips. During narrow-base walking, participants were instructed to adopt a step width smaller than both the width of their hips and their usual step width. Throughout wide- and narrow-base trials, participants received repeated verbal instruction to maintain wider and narrower step widths, respectively, compared to normal walking. Participants walked in each condition for 8 min while being exposed to continuous EVS and were guided by the beat of a metronome at 78 steps/ min to control for effects of varying cadence on vestibular contributions during locomotion ${ }^{11,12}$. The walking speed of $0.8 \mathrm{~m} / \mathrm{s}$ and cadence 78 steps $/ \mathrm{min}$ were chosen to replicate the conditions of Dakin et al. ${ }^{11}$. These walk- 
ing parameters also ensure that vestibular-evoked balance responses, which are known to decrease as velocity and cadence increase $\mathrm{e}^{11,12}$, could be measured throughout the gait cycle.

Prior to starting the experiments, participants were allowed to walk for 3-4 min to familiarize themselves with walking on the treadmill at the specific cadence and with the electrical stimulus. In addition, participants walked for $2 \mathrm{~min}$ in each condition before the electrical stimulus was applied, which together with the familiarization period was considered a sufficient exposure period to remove acclimatization effects of walking on a treadmill ${ }^{50,51}$. Trial order for the different walking conditions was also randomized for each subject and subjects were given a short $5 \mathrm{~min}$ break between trials to limit the influence of any long-term habituation to the electrical stimulus throughout the walking trials ${ }^{52}$. Finally, participants maintained their head in a slightly extended position with the Reid's plane pitched $\sim 18^{\circ}$ up from horizontal ${ }^{40,41}$ by keeping a headgear-mounted laser on a target located $3 \mathrm{~m}$ in front of them. This head position was chosen to maximize the amplitude of vestibulomotor balance responses in the mediolateral direction ${ }^{41,53,54}$.

Instrumentation. Kinematic data were recorded using a 3D motion capture system (Optotrak, Northern Digital Inc., Waterloo, Ontario, Canada) sampling at 100 samples/s. Clusters of three light emitting diodes (LED) were positioned at the occipital lobe, the spinous process of the sixth thoracic vertebra (T6), the posterior superior iliac spine and at the calcaneus bilaterally. Ground reaction forces (GRF) were measured from each belt by force plates embedded in the treadmill (Motekforce Link, The Netherlands) at a sampling rate of 200 samples/s. In addition to analysis of the coupling between EVS and mediolateral forces as described below, these signals were used for the identification of toe-off and heel-strike and gait events.

Surface electromyography (EMG) (TMSI Porti system, TMSI Enschede, the Netherlands) was collected at 2000 samples/s bilaterally from the medial gastrocnemius, gluteus medius and erector spinae muscles, using pairs of disposable self-adhesive $\mathrm{Ag} / \mathrm{AgCl}$ surface electrodes (Ambu, Balerrup, Denmark; model Blue sensor; diameter $30 \times 22 \mathrm{~mm}$ ) for each muscle. Electrodes were placed over the recorded muscles according to SENIAM electrodes placement recommendations ${ }^{55}$ after abrading and cleaning the skin with alcohol. A reference electrode was placed over the medial bony part of the left wrist (styloid process). The three muscles measured were chosen based on their supposed roles in different stabilizing strategies that may be employed throughout the gait cycle and across walking conditions. The medial gastrocnemius muscle (and other ankle plantarflexors) act as the foot's prime movers during normal walking ${ }^{56}$ and are most sensitive to vestibular input in the late stance phase $\mathrm{e}^{11,12,15}$ when modulating push-off force. The gluteus medius muscle contributes to foot placement strategies; its activity is correlated to the next foot placement ${ }^{57,58}$ and is most sensitive to imposed vestibular errors just prior to heel strike $^{11}$. Finally, trunk muscles serve to directly influence trunk motion relative to the pelvis and are primarily activated to stabilize the trunk during weight transfer around heel strike ${ }^{59,60}$. Therefore, erector spinae muscle may be especially suited to contribute to angular momentum control of the torso during narrow base walking ${ }^{61}$ when push-off modulation and foot-placement are rendered ineffective.

Data analysis. Force-plate data were used first to calculate center of pressure positions, which were in turn used to identify heel contacts ${ }^{62}$. From these estimates, stride time was calculated as the duration between two consecutive heel strikes of the same foot and the step width was determined as the mediolateral distance between the centroids of the feet cluster markers at heel strike. These measures of limb kinematics (stride time and step width) were compared across walking conditions to determine whether participants adhered to our instructions (that is, walk at the metronome-guided cadence and with a modified step width). We compared muscle activity across each walking condition after rectifying and low-pass filtering (20 Hz cutoff, zero lag, sixth order Butterworth) the EMG signals and then time-normalizing the data per stride and averaging the data across the 256 strides. Prior to averaging, each rectified and low-pass filtered EMG signal was normalized to the maximum amplitude across all conditions.

To assess changes in local dynamic stability during the different walking conditions, we estimated the local divergence exponent (LDE). Measures of local dynamic stability for walking indicate the ability for a participant to return to the steady-state periodic motion after infinitesimally small perturbations ${ }^{63}$, which occur, for example, through natural variability in the walking surface or the neuromuscular system. These measures have been shown to be particularly useful for detecting patients at risk of falling ${ }^{3}$. The LDE measures the exponential rate of divergence of neighboring trajectories of a state space constructed from kinematic data of gait ${ }^{64}$, whereby an increasing LDE indicates reduced stability. We calculated the LDE using Rosenstein's algorithm ${ }^{65}$ and as input the velocity of a maker placed over the T6 vertebrae, which was estimated using a three-point differentiation of the position trace ${ }^{66}$. Velocity time series were first resampled so that each time series of 256 strides (the minimum number of strides collected from every participant) contained 25,600 samples. The LDE was then calculated as the slope of the mean divergence curve, whose horizontal axis was normalized by stride time from 0-0.5 stride ${ }^{67,68}$.

To examine the vestibulomotor coupling between the input stimulus (i.e. EVS) and motor output (EMG and ground reaction forces) across our conditions, we computed the time-frequency coherence and gain assuming a linear stimulus-response relationship ${ }^{69}$. Prior to estimating coherence and gain, EVS, EMG and ground reaction forces were cut into segments synchronized to heel strikes. Based on the symmetry of our walking conditions, we chose to align the data on each limb's heel strike in order to pool responses from muscles in the left and right limbs. Symmetry of the evoked muscle responses was confirmed prior to pooling the data (see Statistical analysis). This approach, however, was not possible for coherence and gain between EVS and GRFs since in our narrowbase walking condition participants regularly made contact of one limb with the opposing belt such that forces from each limb could not be measured on the separate force plates. Therefore, forces from both plates were first summed before estimating the coherence. To avoid distortion in the coherence estimates at the beginning and end of the signal, each stride was padded with data from the neighboring strides (50\%). EVS and rectified EMG 
were low-pass filtered (100 Hz cutoff, zero lag, sixth-order Butterworth) and down-sampled to 200 samples/s. To account for stride-to-stride variations, stride duration was normalized by resampling the data according to the average stride duration of all trials. This normalization was performed on the auto-spectra of the EVS, EMG and force signals, as well as on their cross-spectra (see below).

Our analysis of coherence and gain was performed based on continuous Morlet wavelet decomposition ${ }^{15,70}$ using Eqs. (1) and (2):

$$
\begin{gathered}
C(\tau, f)=\frac{\left|P_{x y}(\tau, f)\right|^{2}}{P_{x x}(\tau, f) P_{y y}(\tau, f)} \\
G(\tau, f)=\left|\frac{P_{x y}(\tau, f)}{P_{x x}(\tau, f)}\right|
\end{gathered}
$$

where $\operatorname{Pxy}(\tau, f)$ ( $\tau$ and $f$ denote the stride time and frequency, respectively) is the time-dependent cross-spectrum between the EVS and rectified EMG or GRF, and $\operatorname{Pxx}(\tau, f)$ and $\operatorname{Pyy}(\tau, f)$ are the time-dependent auto-spectra of the EVS and rectified EMG or GRF, respectively. Coherence ranges from zero to one, and provides a measure of the linear relationship between two signals ${ }^{71}$ as well as an estimate of their coupling (i.e. shared variance) at each frequency ${ }^{72}$. Because coherence is normalized to the auto-spectra of the input and output signals, it can be sensitive to variation in non-vestibular contributions to the measured output signal (i.e. magnitude changes of EMG or ground reaction forces). Gain on the other hand indicates the magnitude of the output relative to the input and is not normalized by the output signal power spectrum; as a result, it is not expected to change even if non-vestibular input leads to changes in output signal magnitude. A preliminary evaluation of our results indicated that similar to previous studies ${ }^{12,15}$, both coherence and gain followed parallel changes in magnitude and timing across the four walking conditions. This suggests that the modulation in coherence was not simply dependent upon the magnitude of the measured output signals, and as a result, we present only the coherence to describe the modulation of vestibular contributions to mediolateral stability. Finally, we evaluated the coherence between EVS and both EMG and GRFs to estimate muscle-specific vestibular contributions and to provide a net estimate of the vestibular input to ongoing locomotor behavior, respectively.

Statistical analysis. We compared all gait parameters (stride time, step width and local dynamic stability) between the four conditions using one-way repeated measures ANOVAs. Subsequently, we performed planned pairwise comparisons (t-test) between normal walking and each modified walking condition (i.e. normal vs. stabilized; normal vs. narrow and normal vs. wide-base walking). EVS-EMG coherence and EVS-GRF coherence were used to identify the phase-dependent coupling between vestibular stimulation and motor responses throughout the walking cycle. For each participant, coherence was defined as significant for those points in the gait cycle where it exceeded 0.018 , corresponding to $\mathrm{p}<0.01$ (for 256 strides) in view of the bi-dimensional nature of the correlations ${ }^{15}$. To determine whether our walking manipulations modified the EVS-EMG coherence when compared to normal walking, we performed cluster-based permutation tests (paired t-tests, 5000 permutations $)^{73}$ between conditions aimed to identify whether the time-frequency coherence significantly differed from the normal condition. In doing so, we did not disregard non-significant coherence values. Since our initial analysis showed no significant between leg differences in coherence, we averaged coherence values over the two legs.

\section{Results}

Effects of condition on gait parameters, muscle activity and local dynamic stability. To characterize changes in gait across walking trials, we first evaluated the gait parameters, muscle activity and stability measures. During all trials, participants were able to maintain stable upright locomotion while exposed to the stochastic electrical stimulation. Despite walking to the beat of a metronome in all conditions, we found a significant main effect of condition on stride time $\left(F(3,30)=4.14 ; \mathrm{p}=0.014 ; \eta^{2}=0.127\right)$. Pairwise comparisons revealed that stride time increased by approximately $6.12 \pm 15.65 \%(0.09 \pm 0.02 \mathrm{~s} ; \mathrm{p}=0.018)$ during stabilized walking when compared to normal walking (Fig. 1a), but it did not change significantly during either widebase $(\mathrm{p}=0.928)$ or narrow-base walking $(\mathrm{p}=0.155)$. As intended, walking condition significantly affected step width $\left(F(3,30)=73.4 ; p<0.001 ; \eta^{2}=0.786\right.$, see Fig. $\left.1 b\right)$. Consistent with previous results ${ }^{20,23-25}$, pairwise analysis revealed that participants reduced their step width by $65.31 \pm 40 \%(0.18 \pm 0.02 \mathrm{~m} ; \mathrm{p}<0.001)$ during stabilized walking, in spite of no explicit instruction to do so. We also found that participants adhered to the step width instructions during the other two conditions, increasing step width by $30.25 \pm 20 \%(0.08 \pm 0.02 \mathrm{~m} ; \mathrm{p}<0.001)$ during wide-base walking and reducing step width by $38.74 \pm 5 \%(0.11 \pm 0.01 \mathrm{~m} ; \mathrm{p}<0.001)$ during narrow-base walking (Fig. 1b).

As expected, when compared to normal walking, significant differences in mediolateral ground reaction forces $^{30,31,74}$ during all other walking conditions were observed primarily during single-support phases of the gait cycle (see Fig. 2a). Forces decreased when subjects were externally supported or walked with a narrow-base and increased when subjects walked with a wide-base (see Supplementary Figure S1). In the medial gastrocnemius and gluteus medius muscles, peak activity in each muscle occurred during the double stance phase $(\sim 50 \%$ of the stride cycle) and after heel strike ( $\sim 20 \%$ of stride cycle), respectively. EMG responses for these two muscles were for the most part overlapping in all conditions, with only limited significant differences observed during the stance phase for some of the conditions relative to normal walking (see Fig. 2b,c and Supplementary Figure S1). Muscle activity in the erector spinae demonstrated more complex phasic activity throughout the gait 
a

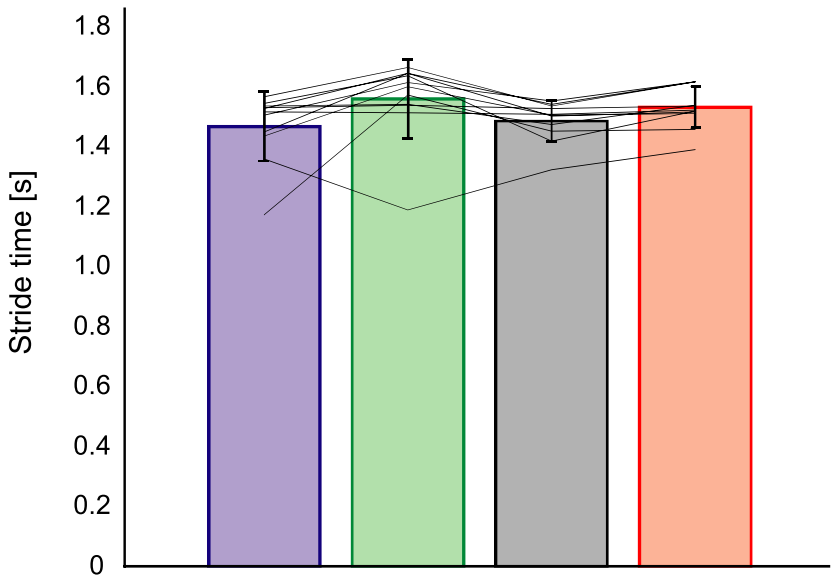

b

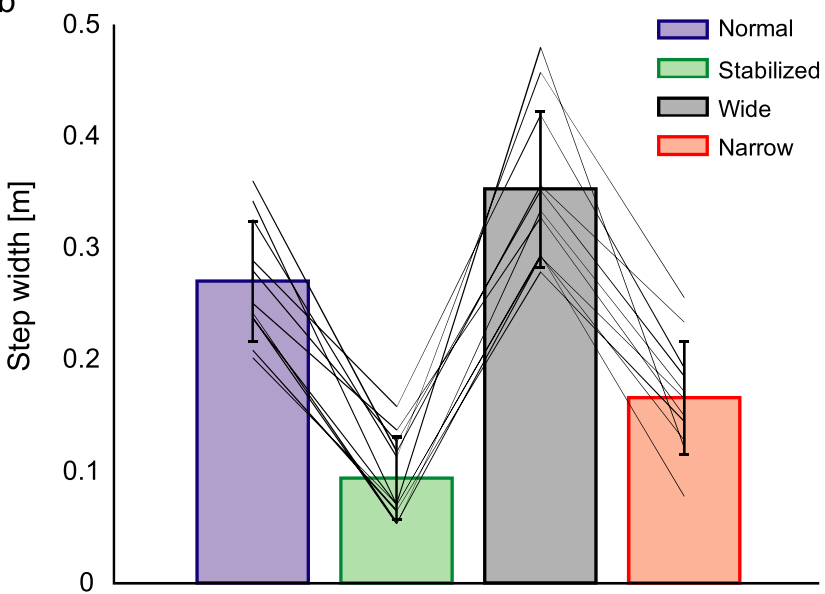

Figure 1. Spatiotemporal parameters of gait during normal walking (blue), walking with external lateral stabilization (green), wide-base walking (black) and narrow-base walking (red). Mean values (bars), standard deviation (error bar) and participants individual changes across conditions (lines) for the stride time (a) and stride width (b) for the four conditions.

cycle with two peaks occurring just after heel strike of each limb. Limited significant differences were observed during stabilized walking and narrow-base walking when compared to normal walking (see Fig. $2 \mathrm{~d}$ and Supplementary Figure S1).

Our manipulations also had a significant effect on mediolateral stability, as expressed by the local divergence exponent $\left(\mathrm{F}(3,30)=129 ; \mathrm{p}<0.001 ; \eta^{2}=0.712\right)$. Pairwise comparisons showed that participants had a higher stability (i.e., lower LDE values) during the stabilized $(\mathrm{p}<0.001)$ and narrow-base walking conditions $(\mathrm{p}=0.019)$ compared to normal walking. Although the mean local divergence exponent was highest (i.e. lowest stability) during wide-base walking, this was not significantly different from normal walking $(\mathrm{p}=0.265)$ (Fig. 3).

EVS-mediolateral GRF and EVS-EMG coherence in normal walking. We next characterized the coupling of the electrical stimulus with both the ground reaction forces and muscle activity in normal walking as a baseline for comparison to our manipulated conditions (Fig. 4-first column). During normal walking, significant EVS-GRF coherence was seen in all participants over the entire gait cycle, with phase-dependent group mean responses that peaked during single stance (Fig. 4 top row). Significant phase-dependent EVS-EMG coupling was also prominent in the mean responses during normal walking, but muscle-specific variations were observed: coherence peaked in mid stance in the medial gastrocnemius, just before heel strike in gluteus medius, and at heel strike and at mid stride (though at a lower magnitude) for the erector spinae. Consistent with previous reports ${ }^{11,12,15}$, peak coherences did not align with peak EMG for any of the muscles, further confirming that vestibular contributions do not depend purely on the excitation of the motoneuron pool. In addition, as commonly observed in vestibular-evoked muscles responses during standing ${ }^{75}$, the bandwidth of significant EVSEMG coherence spanned $\sim 0-25 \mathrm{~Hz}$ while significant EVS-GRF coherence was observed from $\sim 0-10 \mathrm{~Hz}$ (Fig. 4).

Stabilization demands but not dynamic stability modulate EVS-GRF and EMG-EVS coupling. To establish the effects of stabilization demands on vestibulomotor coupling, we examined the difference in coherence between normal walking and all other conditions (Fig. 5). During externally stabilized walking, both EVS-GRF and EVS-EMG coherences decreased significantly relative to normal walking (see Figs. 4 and 5). More specifically, the reduced coupling during the stabilized condition was primarily observed during the periods of peak coherence in normal walking (i.e. single stance for GRF and medial gastrocnemius, and before/at heel strike for gluteus medius and erector spinae). Although the increased stride time (i.e. decreased cadence) during stabilized walking (see Fig. 1) may have acted as a confounding factor to these changes ${ }^{11}$, this effect commonly increases vestibulomotor responses in contrast to the observed decrease in coherence seen here. During wide-base walking, EVS-GRF and EVS-EMG coherences were also significantly decreased compared to normal walking (Fig. 5). The EVS-GRF coherence decreased over the majority of the gait cycle with the most prominent changes observed during the periods of peak coherence seen in the normal condition. Further, EVS-EMG coherence was reduced for all three muscles in wide-base walking, again with the greatest differences observed at instants of peak coherence in normal walking (Fig. 5). Taken together, the results of stabilized and wide-base walking show a reduction in vestibular input to the net muscle activity of the body (i.e. GRFs), which is driven at least in part by the three muscles measured when stabilization demands (but not dynamic stability) are decreased.

During narrow-base walking, we observed more complex changes in coupling between EVS and GRF and between EVS and muscle activity. Figures 4 and 5 together show that EVS-GRF coherence during narrow-base walking significantly increased compared to normal walking over the entire gait cycle. While this was matched by an increase in EVS-EMG coherence in the erector spinae muscle, we found unchanged EVS-EMG coupling in 

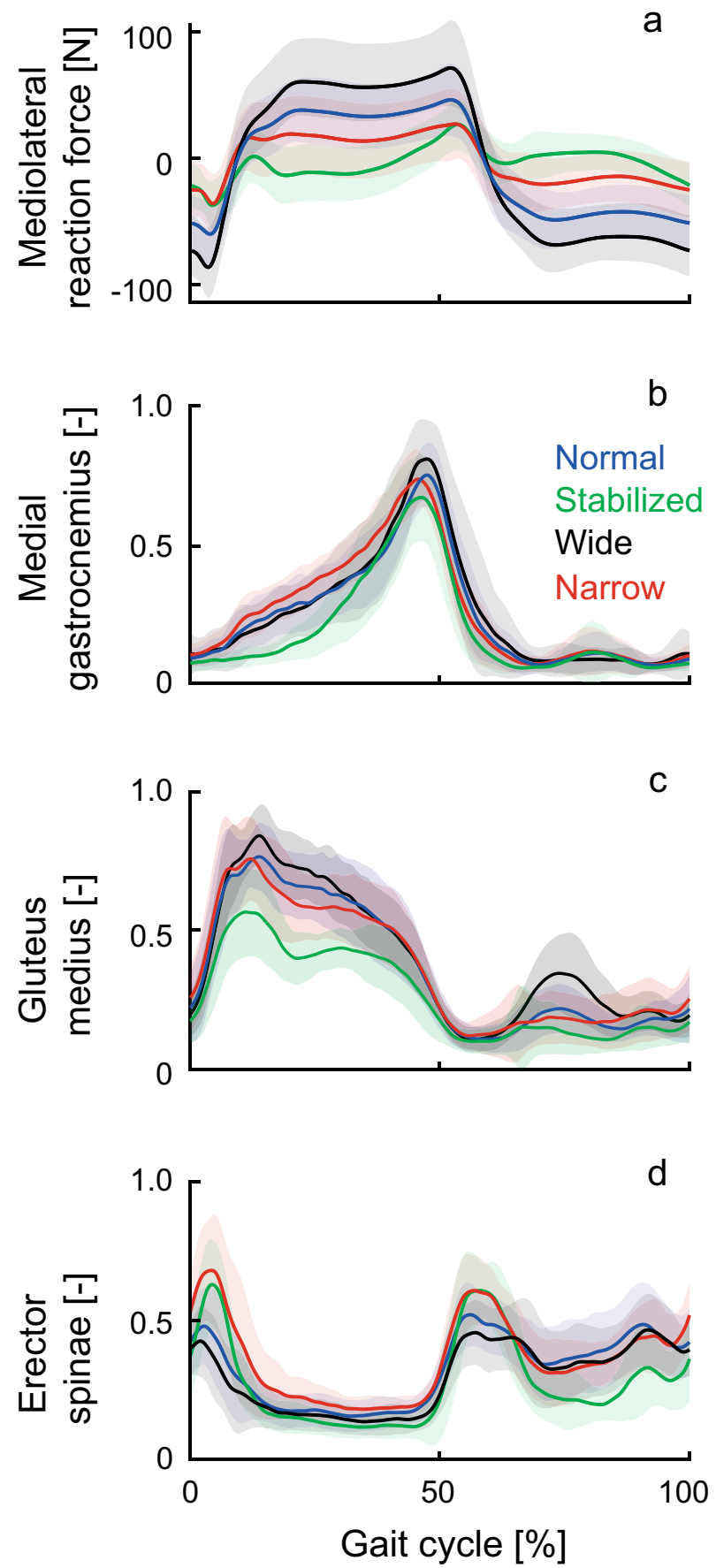

Figure 2. Ground reaction force and electromyographic amplitudes throughout the gait cycle during normal walking (blue line), walking with external lateral stabilization (green line), wide-base walking (black line) and narrow-base walking (red line). Mean values (lines) and standard deviation (shadowed area) of the ground reaction force (a) and the EMG envelopes of the medial gastrocnemius (b), gluteus medius (c) and erector spinae (d) muscles for all conditions. For each muscle, EMG signals were normalized to the maximum amplitude across all conditions.

the gluteus medius muscle and a decrease in coherence in the medial gastrocnemius muscle. These more complex changes in vestibular-evoked motor responses suggest that while the net output of the vestibular-evoked muscle activity (i.e. EVS-GRF coherence) increases with increased stabilization demands, as well as the dynamic stability (see Fig. 3), this trend is not reflected in EVS-EMG coupling of all muscles. 


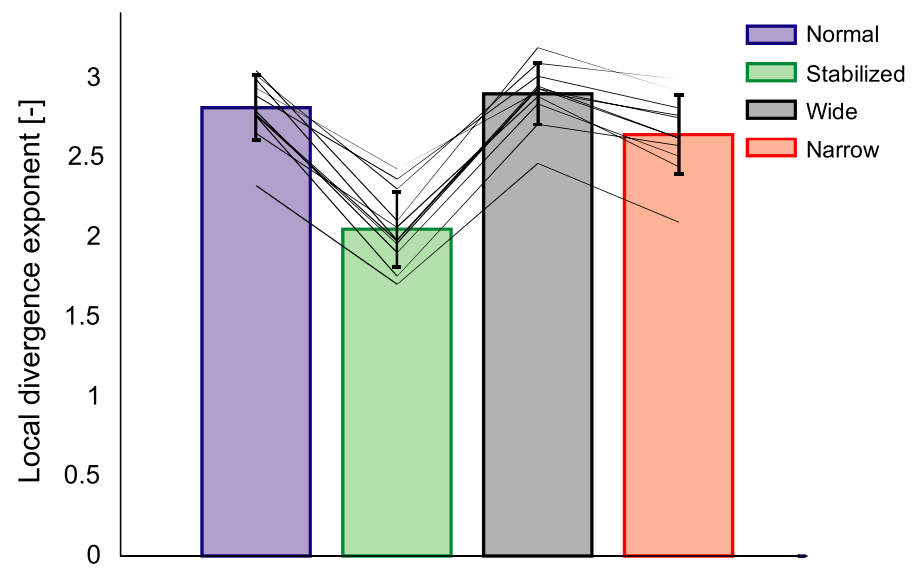

Figure 3. Local divergence exponents during normal walking (blue), walking with external stabilization (green), wide-base walking (black) and narrow-base walking (red). Mean values (bars), standard deviation (error bar) and participants' individual changes across conditions (lines) for the evaluated conditions.
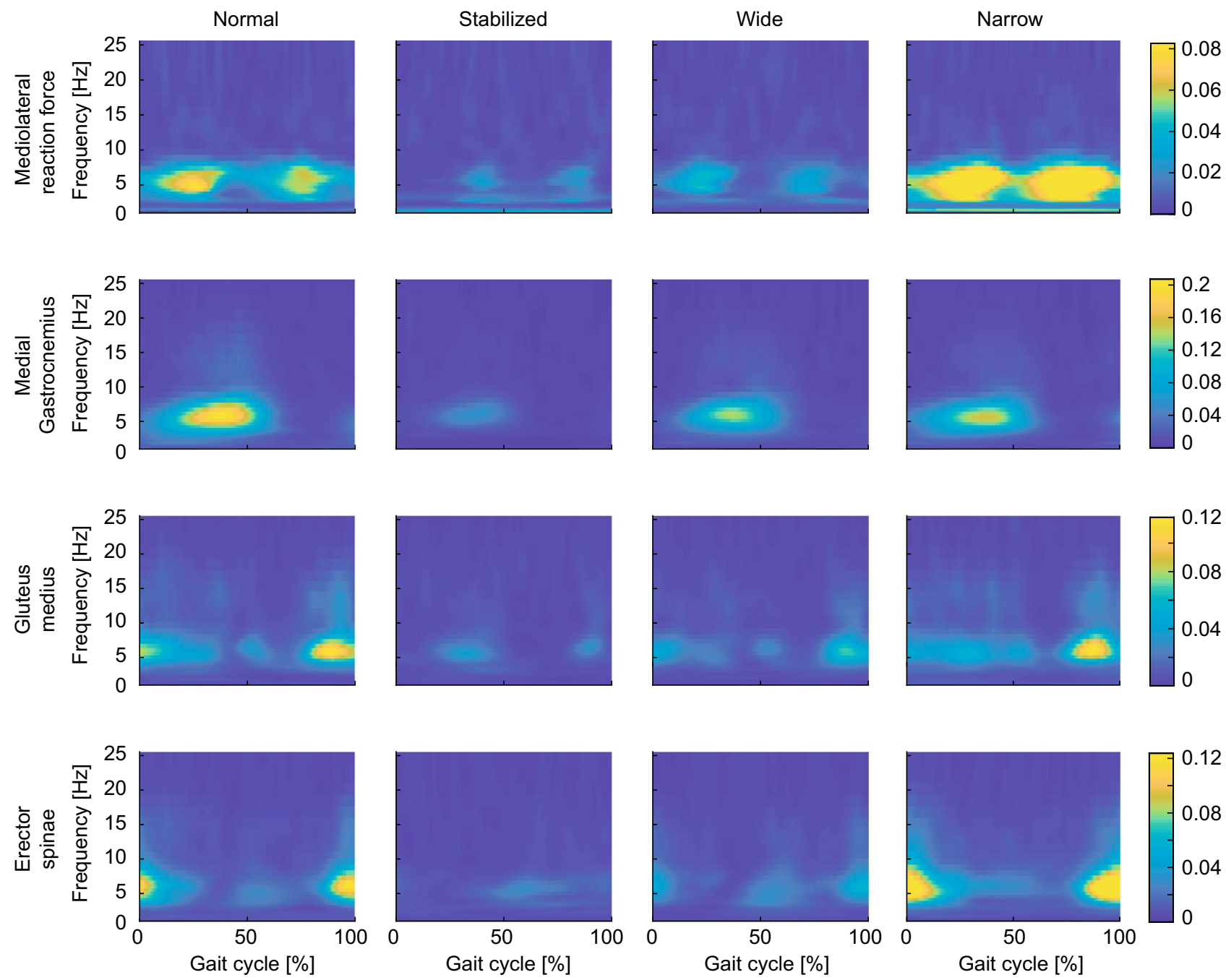

Figure 4. Coherence plots of EVS-GRF (first row) and EVS-EMG for medial gastrocnemius (second row), gluteus medius (third row) and erector spinae (fourth row) for normal walking (first column), walking with external stabilization (second column), wide-base walking (third column) and narrow-base walking (fourth column). Coherence magnitude is indicated by the color bars. 

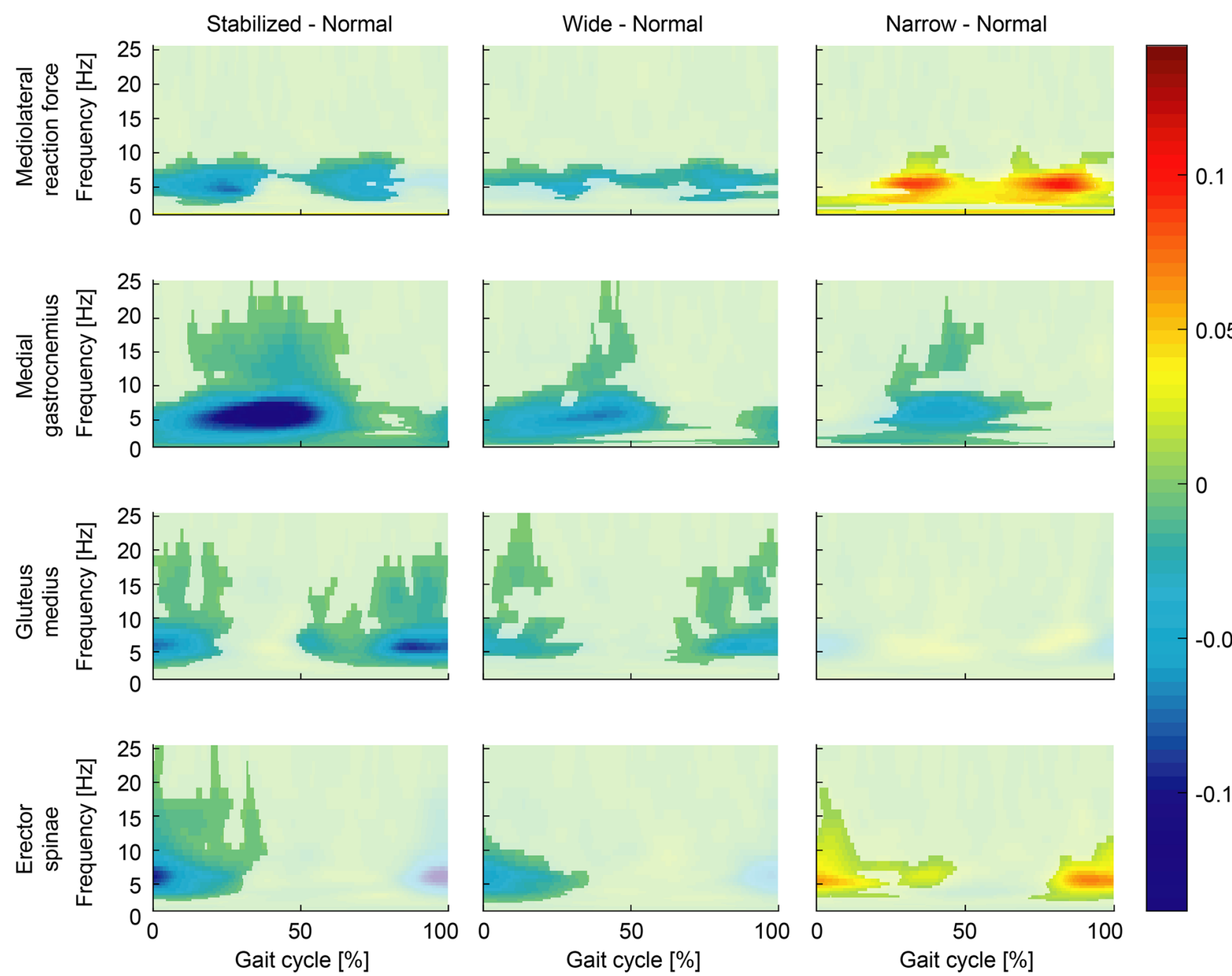

Figure 5. Differences in time-frequency coherence between the normal walking condition and walking with external lateral stabilization (first column), wide-base walking (second column) and narrow-base walking (third column). Color coding refers to the difference in coherence between two walking conditions (e.g. normal minus stabilized) for the EVS-GRF coherence (first row) and EVS-EMG coherence of medial gastrocnemius (second row), gluteus medius (third row) and erector spinae (forth row) muscles. For illustrative purposes, differences that were not significant were plotted slightly opaque.

\section{Discussion}

We characterized how coupling of vestibular input with muscle activity and ground reaction forces modulate as a function of the stabilization demands during locomotion. We found that as participants walked with decreased stabilization demands through either external stabilization or wider step widths, coherence between electrical vestibular stimulation and both muscle activity and ground reaction forces decreased compared to normal walking. These overall reductions in vestibulomotor coupling were accompanied by an increase or no change in the stability of the gait pattern-measured as a decreased or constant local divergence exponent-during stabilized and wide-base walking, respectively. In contrast, the increased stabilization demands of walking with narrow steps invoked complex changes in vestibulo-muscular coupling that increased or decreased specific to each muscle's involvement in correcting for the imposed vestibular error. Nevertheless, these changes in vestibulomuscular coupling increased the collective contribution of vestibular signals to the ground reaction forces and occurred together with a decrease in the local divergence exponent (i.e. increased gait stability). This suggests that participants maintained a more stable gait pattern during narrow walking that was at least partially subserved through increased use of vestibular feedback. Ultimately, these results indicate that vestibular contributions to gait stability may be modulated with frontal plane stability, but that they more specifically depend on the stabilization demands (i.e. control effort) required to maintain a stable gait pattern and not the stability of the gait pattern itself.

When participants walked with external stabilization, the stability of the gait pattern increased (i.e. decreasing LDE) while vestibular-evoked muscle and force responses decreased as compared to normal walking. Both of these results are not entirely surprising since the control of mediolateral motion is aided by the forces generated by the springs $s^{19,20,25}$. As a result, there is a reduced reliance on vestibular signals to maintain upright locomotion during stabilized walking. This is similar to the task dependent reductions in vestibular input observed during 
standing ${ }^{44,45,76-78}$ when participants are externally supported; stimulus-evoked responses are suppressed since the vestibular feedback is no longer relevant to balancing the body. Our results reveal that these task dependent changes in the vestibular control of standing also apply during the more dynamic task of walking. In addition, they also support the proposal that anteroposterior control of whole-body stability during locomotion is controlled passively ${ }^{17}$. By making the body passively stable in the mediolateral direction, we saw a reduction in vestibular-evoked responses that matched the near absence of vestibular contributions when the vestibular error is directed in the anterior-posterior direction ${ }^{16}$.

When participants walked with a wide base, vestibular-evoked muscle and force responses also decreased in a manner that parallels the effects of wide stance during standing ${ }^{44,79}$. Walking (and standing) with a wide foot placement increases the base of support and the passive stiffness in the frontal plane. The current results show that the corrective contribution of vestibular signals during walking with a wide base decrease in a manner similar to the effects seen during external stabilization. Our measure of dynamic stability, however, did not follow the same trend. Instead, we observed a slight (albeit non-significant) increase in the local divergence exponent compared to normal walking. This aligns with previous estimates of a constant or decreased dynamic gait stability during wide-base walking ${ }^{30,34}$. A key difference between stabilized and wide-base walking is that in the former, increased gait stability and upright balance is an inevitable result of the external support. Wide-base walking, on the other hand, despite the increased base of support, still demands active stabilization, and the manner in which this is achieved differs from normal walking. For example, push-off modulation and step-by-step foot placement precision both decrease when walking with wide steps $\mathrm{s}^{30}$. In addition, the increased moment arm of the ground reaction forces about the center of mass generates greater fluctuations in angular plane momentum ${ }^{80}$, which in and of themselves are destabilizing. These changes may be possible because the margins for error of balance control are inherently increased, making wide-base walking more robust to external disturbances. The current results therefore suggest that vestibular contributions may also decrease under walking conditions with reduced control effort to maintain mediolateral stability. This is in line with recent findings in patients with vestibular hypofunction who walk slower, with increased cadence and wider steps ${ }^{81-85}$. Our results suggest that they may adopt these changes in walking behavior to be less dependent on vestibular input.

The possibility that vestibular contributions are modulated with the control effort (i.e. stabilization demand) of gait is further supported by our narrow-base walking results. Both the dynamic stability of gait and vestibularevoked ground reaction forces during narrow-base walking increased relative to normal walking. The increase in dynamic stability (i.e. decreasing LDE) in particular is thought to be required to constrain torso motion to margins of error within which walking with narrow steps can be maintained ${ }^{34}$. Indeed, foot placement during narrow-base walking is more tightly coupled to center-of-mass motion ${ }^{30,31}$ and participants maintain smaller step-to-step oscillations ${ }^{86}$. Our results show that under these highly regulated conditions, this increase in control effort may be driven, at least in part, by a net increase in vestibular input (as reflected in the ground reaction forces). This overall increase in vestibular contribution to mediolateral stability, however, does not seem to originate from a general upregulation of vestibular responses in all muscles. Instead, we found decreasing and unchanging vestibular responses in medial gastrocnemius and gluteus medius muscles, respectively, while vestibular responses in erector spinae muscles increased. These muscle specific changes align with the scaling of evoked responses according to the muscle's involvement in correcting for imposed errors ${ }^{11,26}$. For example, moments generated by the ankle muscles, such as the medial gastrocnemius, strongly contribute to balance corrections through push-off modulation ${ }^{87}$. During narrow-base walking, however, push-off modulation in the mediolateral direction is not a viable option since the push off force has only a minimal moment arm. Similarly, regulation of mediolateral foot placement as provided by the gluteus medius ${ }^{11,57}$ is rendered less effective, since narrow-base walking constrains the foot to a restricted range. Instead, humans more commonly rely on the modulation of the torso's angular moment to maintain upright balance ${ }^{61,86}$. Although the muscles around the ankles and hips also contribute to frontal plane angular momentum during normal walking ${ }^{80}$, the direct influence of spinal muscles on trunk motion may make them more suited to contribute to balance during narrow walking by producing or responding to rapid trunk tilts. A limitation to this interpretation, however, is that we cannot rule out the influence of arm movements, which are known to influence the stability of human gait ${ }^{88-90}$, since we did not measure arm movements or provide specific instructions to participants to control their movement. Nevertheless, our erector spinae results indicate that the influence of spinal muscle activity provides a key, and perhaps primary, contribution to the net vestibular output to ground reaction forces to maintain upright balance during narrow-base walking.

Significant coherence between the electrical stimulus and erector spinae muscle activity in all conditions also demonstrates that the phasic contribution of this particular axial muscle to whole-body mediolateral stability can be flexibly modulated to address varying stabilizing demands. This is similar to this muscle's differential response to vestibular disturbances in standing and sitting ${ }^{91}$. Others have reported, however, that erector spinae muscles, unlike lower limb muscles, maintain a fixed response sensitivity to vestibular input throughout all phases of walking ${ }^{92}$. This follows a similar argument made in neck muscles where vestibulocollic reflexes are maintained regardless of the requirement to maintain head on trunk balance ${ }^{93,94}$. By delivering square wave EVS pulses at and slightly after (15\% of the gait cycle) the heel strike, Guillaud et al. (2020) argued that the invariant response of the muscle to the electrical stimulation at these two time points indicate a fixed vestibular sensitivity throughout the entire gait cycle ${ }^{92}$. The improved resolution of the time-varying techniques used here, however, reveals that this muscle does indeed modulate its response to vestibular input throughout locomotion: EVSEMG coherence peaked at heel strike and dropped to near-zero at approximately $40 \%$ of the stride cycle. The restricted time-window of the two stimulation occurrences considered (i.e. at and slightly after heel strike) in the study from Guillaud et al., however, may have masked any changes in EVS-evoked responses. In addition, the modulation of this muscle's vestibular sensitivity extends across walking conditions, which nearly doubled during narrow-base walking as compared to normal walking. 
A limitation of our study is that while vestibular-evoked responses were quantified throughout the gait cycle, the local divergence exponent remains a mean measure of stability for the entire gait cycle. It may therefore be possible that time-varying changes in gait stability contribute to the phase-dependent vestibular responses seen here. While early studies using phase-dependent metrics of gait stability suggested that these measures may hold promise ${ }^{95,96}$, a recent study from our lab doubted their use, as we found only limited correlation between phasedependent gait stability measures and the probability of falling in a simple dynamic walking model ${ }^{97}$. However, if adequate phase-dependent gait stability measures become available, the relationship between phase-dependent gait stability and phase-dependent vestibular responses may be an interesting topic of study.

In conclusion, we have shown that the muscle and whole-body responses evoked by a vestibular stimulation differ according to the gait stabilization demands. When stability is increased by external support, the muscle and whole-body responses to the vestibular stimulus are substantially reduced. During wide-base walking vestibular-evoked muscle and force responses also decrease, though these changes in vestibular contribution are not accompanied by increased dynamic gait stability. Conversely, narrow-base walking produced complex muscle-specific responses that resulted in an increase in the net vestibular contribution to ground reaction forces and increased stability of gait. Overall, our results show that although the vestibular control of gait stability may vary with frontal plane stability, they critically depend on the stabilization demands (i.e. control effort) needed to maintain stable walking patterns.

Received: 6 October 2020; Accepted: 4 June 2021

Published online: 02 July 2021

\section{References}

1. Bruijn, S. M., Meijer, O. G., Beek, P. J. \& van Dieen, J. H. Assessing the stability of human locomotion: A review of current measures. J. R. Soc. Interface 10, 20120999. https://doi.org/10.1098/rsif.2012.0999 (2013).

2. van Emmerik, R. E. A., Ducharme, S. W., Amado, A. C. \& Hamill, J. Comparing dynamical systems concepts and techniques for biomechanical analysis. J. Sport Health Sci. 5, 3-13. https://doi.org/10.1016/j.jshs.2016.01.013 (2016).

3. Mehdizadeh, S. The largest Lyapunov exponent of gait in young and elderly individuals: A systematic review. Gait Posture 60, 241-250. https://doi.org/10.1016/j.gaitpost.2017.12.016 (2018).

4. Misiaszek, J. Neural control of walking balance: If falling then react else continue. Exerc. Sport Sci. Rev. 34, 128-134 (2006).

5. Chien, J. H., Mukherjee, M. \& Stergiou, N. Mastoid vibration affects dynamic postural control during gait. Ann. Biomed. Eng. 44, 2774-2784. https://doi.org/10.1007/s10439-016-1556-z (2016).

6. Stergiou, N. \& Decker, L. M. Human movement variability, nonlinear dynamics, and pathology: Is there a connection?. Hum. Mov. Sci. 30, 869-888. https://doi.org/10.1016/j.humov.2011.06.002 (2011).

7. Rossignol, S., Dubuc, R. \& Gossard, J. P. Dynamic sensorimotor interactions in locomotion. Physiol. Rev. 86, 89-154. https://doi. org/10.1152/physrev.00028.2005 (2006).

8. Cullen, K. E. The vestibular system: Multimodal integration and encoding of self-motion for motor control. Trends Neurosci. 35, 185-196. https://doi.org/10.1016/j.tins.2011.12.001 (2012).

9. Brandt, T. Vestibulopathic gait: You're better off running than walking. Curr. Opin. Neurol. 13, 3-5 (2000).

10. Borel, L. et al. Walking performance of vestibular-defective patients before and after unilateral vestibular neurotomy. Behav. Brain Res. 150, 191-200 (2004).

11. Dakin, C. J., Inglis, J. T., Chua, R. \& Blouin, J. S. Muscle-specific modulation of vestibular reflexes with increased locomotor velocity and cadence. J. Neurophysiol. 110, 86-94. https://doi.org/10.1152/jn.00843.2012 (2013).

12. Forbes, P. A. et al. Rapid limb-specific modulation of vestibular contributions to ankle muscle activity during locomotion. J. Physiol. 595, 2175-2195. https://doi.org/10.1113/JP272614 (2017).

13. Bent, L. R., Inglis, J. T., Bradford, J. \& Mcfadyen, B. J. When is vestibular information important during walking?. J. Neurophysiol. 92, 1269-1275 (2004).

14. Iles, J. F., Baderin, R., Tanner, R. \& Simon, A. Human standing and walking: Comparison of the effects of stimulation of the vestibular system. Exp. Brain Res. 178, 151-166. https://doi.org/10.1007/s00221-006-0721-2 (2007).

15. Blouin, J. S. et al. Extracting phase-dependent human vestibular reflexes during locomotion using both time and frequency correlation approaches. J. Appl. Physiol. 1985(111), 1484-1490. https://doi.org/10.1152/japplphysiol.00621.2011 (2011).

16. Tisserand, R. et al. Down regulation of vestibular balance stabilising mechanisms to enable transition between motor states. Elife https://doi.org/10.7554/eLife.36123 (2018).

17. Bauby, C. E. \& Kuo, A. D. Active control of lateral balance in human walking. J. Biomech. 33, 1433-1440 (2000).

18. McGeer, T. Passive dynamic walking. Int. J. Robot. Res. T 9, 62-82. https://doi.org/10.1177/027836499000900206 (1990).

19. Mahaki, M., Bruijn, S. M. \& van Dieen, J. H. The effect of external lateral stabilization on the use of foot placement to control mediolateral stability in walking and running. PeerJ 7, e7939. https://doi.org/10.7717/peerj.7939 (2019).

20. Donelan, J. M., Shipman, D. W., Kram, R. \& Kuo, A. D. Mechanical and metabolic requirements for active lateral stabilization in human walking. J. Biomech. 37, 827-835. https://doi.org/10.1016/j.jbiomech.2003.06.002 (2004).

21. Ortega, J. D., Fehlman, L. A. \& Farley, C. T. Effects of aging and arm swing on the metabolic cost of stability in human walking. J. Biomech. 41, 3303-3308. https://doi.org/10.1016/j.jbiomech.2008.06.039 (2008).

22. Dean, J. C., Alexander, N. B. \& Kuo, A. D. The effect of lateral stabilization on walking in young and old adults. IEEE Trans. Biomed. Eng. 54, 1919-1926 (2007).

23. Ijmker, T., Houdijk, H., Lamoth, C. J., Beek, P. J. \& van der Woude, L. H. Energy cost of balance control during walking decreases with external stabilizer stiffness independent of walking speed. J. Biomech. 46, 2109-2114. https://doi.org/10.1016/j.jbiomech. 2013.07.005 (2013).

24. Ijmker, T. et al. Can external lateral stabilization reduce the energy cost of walking in persons with a lower limb amputation?. Gait Posture 40, 616-621. https://doi.org/10.1016/j.gaitpost.2014.07.013 (2014).

25. Bruijn, S. M., Van Dieen, J. H. \& Daffertshofer, A. Beta activity in the premotor cortex is increased during stabilized as compared to normal walking. Front. Hum. Neurosci. 9, 593. https://doi.org/10.3389/fnhum.2015.00593 (2015).

26. Forbes, P. A., Luu, B. L. \& Blouin, J. S. Transformation of vestibular signals for the control of standing in humans. J. Neurosci. 36, 11510-11520. https://doi.org/10.1523/JNEUROSCI.1902-16.2016 (2016).

27. Kubinski, S. N., McQueen, C. A., Sittloh, K. A. \& Dean, J. C. Walking with wider steps increases stance phase gluteus medius activity. Gait Posture 41, 130-135. https://doi.org/10.1016/j.gaitpost.2014.09.013 (2015).

28. Aboutorabi, A., Arazpour, M. \& Bahramizadeh, M. The effect of aging on gait parameters in able-bodied older subjects: A literature review. Aging Clin. Exp. Res. 28, 393-405 (2016). 
29. Schrager, M. A., Kelly, V. E., Price, R., Ferrucci, L. \& Shumway-Cook, A. The effects of age on medio-lateral stability during normal and narrow base walking. Gait Posture 28, 466-471. https://doi.org/10.1016/j.gaitpost.2008.02.009 (2008).

30. Perry, J. A. \& Srinivasan, M. Walking with wider steps changes foot placement control, increases kinematic variability and does not improve linear stability. R. Soc. Open Sci. 4, 160627. https://doi.org/10.1098/rsos.160627 (2017).

31. Arvin, M. et al. Effects of narrow base gait on mediolateral balance control in young and older adults. J. Biomech. 49, 1264-1267. https://doi.org/10.1016/j.jbiomech.2016.03.011 (2016).

32. Hak, L. et al. Speeding up or slowing down? Gait adaptations to preserve gait stability in response to balance perturbations. Gait Posture 36, 260-264. https://doi.org/10.1016/j.gaitpost.2012.03.005 (2012).

33. Young, P. M. M. \& Dingwell, J. B. Voluntary changes in step width and step length during human walking affect dynamic margins of stability. Gait Posture 36, 219-224. https://doi.org/10.1016/.j.gaitpost.2012.02.020 (2012).

34. Young, P. M. M. \& Dingwell, J. B. Voluntarily changing step length or step width affects dynamic stability of human walking. Gait Posture 35, 472-477. https://doi.org/10.1016/j.gaitpost.2011.11.010 (2012).

35. Murray, A. J., Croce, K., Belton, T., Akay, T. \& Jessell, T. M. Balance control mediated by vestibular circuits directing lim extension or antagonist muscle co-activation. Cell Rep. 22, 1325-1338. https://doi.org/10.1016/j.celrep.2018.01.009 (2018).

36. Goldberg, J. M., Fernandez, C. \& Smith, C. E. Responses of vestibular-nerve afferents in the squirrel monkey to externally applied galvanic currents. Brain Res. 252, 156-160 (1982).

37. Kim, J. \& Curthoys, I. S. Responses of primary vestibular neurons to galvanic vestibular stimulation (GVS) in the anaesthetised guinea pig. Brain Res. 64, 265-271. https://doi.org/10.1016/j.brainresbull.2004.07.008 (2004).

38. Kwan, A., Forbes, P. A., Mitchell, D. E., Blouin, J. S. \& Cullen, K. E. Neural substrates, dynamics and thresholds of galvanic vestibular stimulation in the behaving primate. Nat. Commun. 10, 1904. https://doi.org/10.1038/s41467-019-09738-1 (2019).

39. Peters, R. M., Rasman, B. G., Inglis, J. T. \& Blouin, J. S. Gain and phase of perceived virtual rotation evoked by electrical vestibular stimuli. J. Neurophysiol. 114, 264-273. https://doi.org/10.1152/jn.00114.2015 (2015).

40. Khosravi-Hashemi, N., Forbes, P. A., Dakin, C. J. \& Blouin, J. S. Virtual signals of head rotation induce gravity-dependent inferences of linear acceleration. J. Physiol. 597, 5231-5246. https://doi.org/10.1113/jp278642 (2019).

41. Fitzpatrick, R. C. \& Day, B. L. Probing the human vestibular system with galvanic stimulation. J. Appl. Physiol. 1985(96), 2301-2316 (2004).

42. St George, R. J. \& Fitzpatrick, R. C. The sense of self-motion, orientation and balance explored by vestibular stimulation. J. Physiol. 589, 807-813. https://doi.org/10.1113/jphysiol.2010.197665 (2011).

43. Nashner, L. M. \& Wolfson, P. Influence of head position and proprioceptive cues on short latency postural reflexes evoked by galvanic stimulation of the human labyrinth. Brain Res. 67, 255-268. https://doi.org/10.1016/0006-8993(74)90276-5 (1974).

44. Mian, O. S. \& Day, B. L. Violation of the craniocentricity principle for vestibularly evoked balance responses under conditions of anisotropic stability. J. Neurosci. 34, 7696-7703. https://doi.org/10.1523/JNEUROSCI.0733-14.2014 (2014).

45. Britton, T. C. et al. Postural electromyographic responses in the arm and leg following galvanic vestibular stimulation in man. Exp. Brain Res. 94, 143-151. https://doi.org/10.1007/BF00230477 (1993).

46. Lund, S. \& Broberg, C. Effects of different head positions on postural sway in man induced by a reproducible vestibular error signal. Acta Physiol. Scand. 117, 307-309. https://doi.org/10.1111/j.1748-1716.1983.tb07212.x (1983).

47. Dakin, C. J., Luu, B. L., van den Doel, K., Inglis, J. T. \& Blouin, J. S. Frequency-specific modulation of vestibular-evoked sway responses in humans. J. Neurophysiol. 103, 1048-1056. https://doi.org/10.1152/jn.00881.2009 (2010).

48. Mahaki, M., Bruijn, S. M. \& van Dieën, J. H. The effect of external lateral stabilization on the control of mediolateral stability in walking and running. PeerJ https://doi.org/10.7287/peerj.preprints.27244v1 (2018).

49. Mahaki, M., IJmker, T., Houdijk, H. \& Bruijn, S. M. How does external lateral stabilization constrain normal gait, apart from improving medio-lateral gait stability? R. Soc open sci. 8, 202088. http://doi.org/10.1098/rsos.202088 (2021).

50. Matsas, A., Taylor, N. \& McBurney, H. Knee joint kinematics from familiarised treadmill walking can be generalised to overground walking in young unimpaired subjects. Gait Posture 11, 46-53. https://doi.org/10.1016/s0966-6362(99)00048-x (2000).

51. Meyer, C. et al. Familiarization with treadmill walking: How much is enough?. Sci. Rep. 9, 5232. https://doi.org/10.1038/s41598019-41721-0 (2019).

52. Hannan, K. B., Todd, M. K., Pearson, N. J., Forbes, P. A. \& Dakin, C. J. Vestibular attenuation to random-waveform galvanic vestibular stimulation during standing and treadmill walking. Sci. Rep. https://doi.org/10.1038/s41598-021-87485-4 (2021).

53. Cathers, I., Day, B. L. \& Fitzpatrick, R. C. Otolith and canal reflexes in human standing. J. Physiol. 563, 229-234. https://doi.org/ 10.1113/jphysiol.2004.079525 (2005).

54. Fitzpatrick, R. C., Butler, J. E. \& Day, B. L. Resolving head rotation for human bipedalism. Curr. Biol. 16, 1509-1514. https://doi. org/10.1016/j.cub.2006.05.063 (2006).

55. Hermens, H. J. et al. SENIAM 8: European recommendations for surface electromyography., Vol. 1 (Roessingh Research and Development, 1999).

56. Liu, M. Q., Anderson, F. C., Schwartz, M. H. \& Delp, S. L. Muscle contributions to support and progression over a range of walking speeds. J. Biomech. 41, 3243-3252. https://doi.org/10.1016/j.jbiomech.2008.07.031 (2008).

57. Rankin, B. L., Buffo, S. K. \& Dean, J. C. A neuromechanical strategy for mediolateral foot placement in walking humans. J. Neurophysiol. 112, 374-383. https://doi.org/10.1152/jn.00138.2014 (2014).

58. van Leeuwen, A. M., van Dieën, J. H., Daffertshofer, A. \& Bruijn, S. M. Active foot placement control ensures stable gait: Effect of constraints on foot placement and ankle moments. PLoS ONE 15(2), e0242215. https://doi.org/10.1371/journal.pone.0242215 (2020)

59. Waters, R. L. \& Morris, J. M. Electrical activity of muscles of the trunk during walking. J. Anat. 111, 191-199 (1972).

60. Thorstensson, A., Carlson, H., Zomlefer, M. R. \& Nilsson, J. Lumbar back muscle activity in relation to trunk movements during locomotion in man. Acta Physiol. Scand. 116, 13-20. https://doi.org/10.1111/j.1748-1716.1982.tb10593.x (1982).

61. Best, A. N. \& Wu, A. R. Upper body and ankle strategies compensate for reduced lateral stability at very slow walking speeds. Proc. R. Soc. B. 287, 20201685. http://doi.org/10.1098/rspb.2020.1685 (2020)

62. Roerdink, M. Online gait event detection using a large force platform embedded in a treadmill. J. Biomech. 41, 2628-2632 (2008).

63. Dingwell, J. B. \& Cusumano, J. P. Nonlinear time series analysis of normal and pathological human walking. Chaos 10, 848-863. https://doi.org/10.1063/1.1324008 (2000).

64. Dingwell, J. B. \& Marin, L. C. Kinematic variability and local dynamic stability of upper body motions when walking at different speeds. J. Biomech. 39, 444-452. https://doi.org/10.1016/j.jbiomech.2004.12.014 (2006).

65. Rosenstein, M. T., Coliins, J. J. \& De Luca, C. J. A practical method for calculating largest Lyapunov exponents from small data sets. Physica 65, 117-134 (1993).

66. Kang, H. G. \& Dingwell, J. B. Dynamic stability of superior vs. inferior segments during walking in young and older adults. Gait Posture 30, 260-263 (2009).

67. Stenum, J., Bruijn, S. M. \& Jensen, B. R. The effect of walking speed on local dynamic stability is sensitive to calculation methods. J. Biomech. 47, 3776-3779. https://doi.org/10.1016/j.jbiomech.2014.09.020 (2014).

68. Bruijn, S.M. Local Dynamic Stability. Zenodo http://doi.org/10.5281/zenodo.573285 (2017).

69. Forbes, P. A. et al. Electrical vestibular stimuli to enhance vestibulo-motor output and improve subject comfort. PLoS ONE $\mathbf{9}$, e84385. https://doi.org/10.1371/journal.pone.0084385 (2014). 
70. Zhan, Y., Halliday, D. W., Jiang, P., Liu, X. \& Feng, J. Detecting time- dependent coherence between non-stationary electrophysiological signals. A combined statistical and time-frequency approach. J. Neurosci. Methods 156, 322-332 (2006).

71. Pintelon, R. \& Schoukens, J. System identification: a frequency domain approach. 2nd edition edn, (Hoboken: John Wiley \& Sons Inc., 2012).

72. Warner, R. M. Spectral Analysis of Time-Series Data. 1 edn, (The Guilford Press, 1998).

73. Maris, E., Schoffelen, J. M. \& Fries, P. Nonparametric statistical testing of coherence differences. J. Neurosci. Methods 163, 161-175. https://doi.org/10.1016/j.jneumeth.2007.02.011 (2007).

74. Hof, A. L., van Bockel, R. M., Schoppen, T. \& Postema, K. Control of lateral balance in walking. Experimental findings in normal subjects and above-knee amputees. Gait Posture 25, 250-258 (2007).

75. Dakin, C. J., Son, G. M., Inglis, J. T. \& Blouin, J. S. Frequency response of human vestibular reflexes characterized by stochastic stimuli. J. Physiol. 583, 1117-1127. https://doi.org/10.1113/jphysiol.2007.133264 (2007).

76. Fitzpatrick, R. C. \& McCloskey, D. I. Proprioceptive, visual and vestibular thresholds for the perception ofsway during standing in humans. J. Physiol. 478, 173-177 (1994).

77. Forbes, P. A., Siegmund, G. P., Schouten, A. C. \& Blouin, J.-S. Task, muscle and frequency dependent vestibular control of posture. Front. Integr. Neurosci. https://doi.org/10.3389/fnint.2014.00094 (2015).

78. Luu, B. L. et al. Human standing is modified by an unconscious integration of congruent sensory and motor signals. J. Physiol. 590, 5783-5794. https://doi.org/10.1113/jphysiol.2012.230334 (2012).

79. Day, B. L., Cauquil, A. S., Bartolomei, M. \& Pastor, M. A. Human body-segmenttiltsinducedbygalvanicstimulation: Avestibularlydrivenbalanceprotectionmechanism. J. Physiol. 500, 661-672 (1997).

80. Neptune, R. R. \& McGowan, C. P. Muscle contributions to frontal plane angular momentum during walking. J. Biomech. 49, 2975-2981. https://doi.org/10.1016/j.jbiomech.2016.07.016 (2016).

81. Liu, P. et al. Characterizing patients with unilateral vestibular hypofunction using kinematic variability and local dynamic stability during treadmill walking. Behav. Neurol. 2017, 4820428. https://doi.org/10.1155/2017/4820428 (2017).

82. Schniepp, R. et al. Locomotion speed determines gait variability in cerebellar ataxia and vestibular failure. Mov. Disord. 27, 125-131. https://doi.org/10.1002/mds.23978 (2012).

83. Gimmon, Y., Millar, J., Pak, R., Liu, E. \& Schubert, M. C. Central not peripheral vestibular processing impairs gait coordination. Exp. Brain Res. 235, 3345-3355. https://doi.org/10.1007/s00221-017-5061-x (2017).

84. Coelho, A. R., Fontes, R. C., Moraes, R., Barros, C. G. C. \& Abreu, D. C. C. Effects of the use of anchor systems in the rehabilitation of dynamic balance and gait in individuals with chronic dizziness of peripheral vestibular origin: A single-blinded, randomized, controlled clinical trial. Arch. Phys. Med. Rehabil. 101, 249-257. https://doi.org/10.1016/j.apmr.2019.07.012 (2020).

85. Herssens, N. et al. An exploratory investigation on spatiotemporal parameters, margins of stability, and their interaction in bilateral vestibulopathy. Sci. Rep. 11, 6427 (2021).

86. Reimann, H., Fettrow, T. \& Jeka, J. J. Strategies for the control of balance during locomotion. Kinesiol. Rev. 7, 18-25. https://doi. org/10.1123/kr.2017-0053 (2018).

87. Kim, M. \& Collins, S. H. Stabilization of a three-dimensional limit cycle walking model through step-to-step ankle control. IEEE Int Conference on Rehabilitation Robotics 24 (2013).

88. Bruijn, S. M., Meijer, O. G., Beek, P. J. \& van Dieen, J. H. The effects of arm swing on human gait stability. J. Exp. Biol. 213, 3945-3952. https://doi.org/10.1242/jeb.045112 (2010).

89. Collins, S. H., Adamczyk, P. G. \& Kuo, A. D. Dynamic arm swinging in human walking. Proc. R. Soc. 276, 3679-3688 (2009).

90. Pijnappels, M., Kingma, I., Wezenberg, D., Reurink, G. \& van Dieën, J. H. Armed against falls: The contribution of arm movements to balance recovery after tripping. Exp. Brain Res. 201, 689-699. https://doi.org/10.1007/s00221-009-2088-7 (2010).

91. Ali, A. S., Rowen, K. A. \& Iles, J. F. Vestibular actions on back and lower limb muscles during postural tasks in man. J. Physiol. 546(2), 615-624. https://doi.org/10.1113/jphysiol.2002.030031 (2003).

92. Guillaud, E. et al. Ancestral persistence of vestibulospinal reflexes in axial muscles in humans. J. Neurophysiol. 123, $2010-2023$. https://doi.org/10.1152/jn.00421.2019 (2020).

93. Forbes, P. A., Siegmund, G. P., Happee, R., Schouten, A. C. \& Blouin, J. S. Vestibulocollic reflexes in the absence of head postural control. J Neurophysiol. 112, 1692-1702. https://doi.org/10.1152/jn.00343.2014 (2014).

94. Forbes, P. A., Fice, J. B., Siegmund, G. P. \& Blouin, J. S. Electrical vestibular stimuli evoke robust muscle activity in deep and superficial neck muscles in humans. Front. Neurol. https://doi.org/10.3389/fneur.2018.00535 (2018).

95. Ihlen, E. A. et al. Phase-dependent changes in local dynamic stability of human gait. J. Biomech. 45, 2208-2214. https://doi.org/ 10.1016/j.jbiomech.2012.06.022 (2012)

96. Mahmoudian, A. et al. Phase-dependent changes in local dynamic stability during walking in elderly with and without knee osteoarthritis. J. Biomech. 49, 80-86. https://doi.org/10.1016/j.jbiomech.2015.11.018 (2016).

97. Jin, J., Kistemaker, D., van Dieën, J. H., Daffertshofer, A. \& Bruijn, S. M. The validation of new phase-dependent gait stability measures: a modeling approach. R. Soc. open sci. 8, 201122. http://doi.org/10.1098/rsos.201122 (2021)

\section{Acknowledgements}

RMM was funded by CAPES (PDSE 19/2016). SMB was funded by a VIDI grant (016.Vidi.178.014) from the Netherlands Organization for Scientific Research (NWO). PAF received funding from the Netherlands Organization for Scientific Research (NWO \#016.Veni.188.049).

\section{Author contributions}

J.V.D. and P.A.F. contributed to the conception or design of the work. R.M.M., S.M.B. and P.A.F. contributed to the acquisition, analysis, or interpretation of data. S.M.B. and P.A.F. made the figures. All authors wrote the main manuscript text, reviewed the manuscript and have approved the submitted version.

\section{Competing interests}

The authors declare no competing interests.

\section{Additional information}

Supplementary Information The online version contains supplementary material available at https://doi.org/ 10.1038/s41598-021-93037-7.

Correspondence and requests for materials should be addressed to P.A.F.

Reprints and permissions information is available at www.nature.com/reprints. 
Publisher's note Springer Nature remains neutral with regard to jurisdictional claims in published maps and institutional affiliations.

(c) (i) Open Access This article is licensed under a Creative Commons Attribution 4.0 International License, which permits use, sharing, adaptation, distribution and reproduction in any medium or format, as long as you give appropriate credit to the original author(s) and the source, provide a link to the Creative Commons licence, and indicate if changes were made. The images or other third party material in this article are included in the article's Creative Commons licence, unless indicated otherwise in a credit line to the material. If material is not included in the article's Creative Commons licence and your intended use is not permitted by statutory regulation or exceeds the permitted use, you will need to obtain permission directly from the copyright holder. To view a copy of this licence, visit http://creativecommons.org/licenses/by/4.0/.

(C) The Author(s) 2021 